\title{
Luminescence of silicon crystallites
}

\author{
C. DELERUE, E. MARTIN, J.-F. LAMPIN, G. ALLAN and M. LANNOO
}

Institut d'Electronique et de Microélectronique du Nord, U.M.R. CNRS 9929, Département ISEN, 41 boulevard Vauban, 59046 Lille cedex, France

The physical origin of the red and infrared light emissions from porous silicon are examined. The red band can come from the direct radiative recombination of excitons in silicon crystallites. The infrared emission can be explained by their recombination on silicon dangling bonds. Finally, the influence of the exchange and valley-orbit splittings on the photoluminescence lifetime is analyzed.

The discovery of the visible luminescence of porous silicon has stimulated many studies concerning excitons in silicon nanocrystallites [1]. To our knowledge, porous silicon is at the origin of three emissions of light under optical excitation. The first one is an intense emission in the visible range [2] $(\sim 1.4$ $\mathrm{eV}-2.2 \mathrm{eV}$ ) which we will call the "red emission" in the following. It is characterized by a long lifetime, between $10 \mu \mathrm{s}$ and $1 \mathrm{~ms}$ depending on the energy of the light. The second emission is in the infrared and seems to be correlated with the existence of dangling bond states [3]. The third one mostly appears in very oxidized porous silicon, is blue and is quite fast [4] (luminescence lifetime of about 1-10 ns). If it is now admitted that the quantum confinement can explain the observed red emission, it is not clear if it can explain the other bands. The aim of our work is to show that the intrinsic excitonic luminescence of silicon crystallites is coherent with the red emission and that the presence of dangling bonds in these crystallites explains the infrared band. The blue emission could be caused by chains of silicon atoms or $\mathrm{Si}$ defects in silicon oxide as shown in separate papers [5]. So we will not discuss further the origins of this blue band and we will concentrate here on the red and infrared emissions. We will also present preliminary results concerning the low temperature dependence of the red luminescence intensity which is influenced by the exchange and valley-orbit splittings in the lowest exciton states.

We have calculated the electronic and optical properties of silicon crystallites. The crystallites have a spherical shape and the same lattice structure than bulk silicon. The dangling bonds at the surface are saturated by hydrogen atoms. We have used the linear combination of atomic orbitals technique (LCAO). Each silicon atom is represented by one $s$ and three $p$ atomic orbitals. The Hamiltonian and overlap matrices are built using the procedure of ref. [6]. Such a LCAO technique gives valence bands and conduction bands in very good agreement with pseudopotential calculations. In the case where we are interested in the influence of a dangling bond state at the surface, one hydrogen atom is removed from the surface. For the studies of the optical properties, we calculate the lifetime of an electron-hole pair in the crystallites. We suppose that the electron and the hole are in a pseudo thermal equilibrium in respectively the conduction and the valence bands and therefore are distributed following a Boltzmann statistics. The rate of recombination $\left(=1 / \tau\right.$ where $\tau$ is the lifetime) from a state $\left|i_{B C}\right\rangle$ of the conduction band to a state $\left|f_{B V}\right\rangle$ of the valence band is estimated using the following law deduced from the Fermi Golden rule:

$$
\frac{1}{\tau}=\frac{16 \pi^{2}}{3} n \frac{e^{2}}{h^{2} m^{2} c^{3}} E_{0}\left|\left\langle i_{B C}|p| f_{B V}\right\rangle\right|^{2}
$$

where $E_{0}$ is the energy of the transition and $n$ is the refraction index (we take $n=1.33$ corresponding to a porous silicon sample of $74 \%$ porosity). The matrix elements in eqn. (1) are evaluated following 
ref. [6]. Eqn. (1) gives the radiative lifetime calculated for a first order process, i.e. which does not involve the mediation of phonons. Since crystalline silicon has an indirect bandgap, this lifetime is infinite in the case of an infinite crystal. Anyway, due to confinement, it is expected that $k$ states mix together giving first order transitions allowed.

The first step of our calculation concerns the electronic structure of silicon crystallites. In particular, we have obtained the dependence of the bandgap with respect to the crystallite diameter. Results have already been published [6] and will not be reproduced here. We show that the red band of porous silicon $(\sim 1.4 \mathrm{eV}-2.2 \mathrm{eV})$ could come from electron-hole recombinations in crystallites with diameter between $2.5 \mathrm{~nm}$ and $4.5 \mathrm{~nm}$. This is in agreement with structural studies of porous silicon. The calculated radiative recombination rates at $\mathrm{T}=300 \mathrm{~K}$ are plotted on Fig. 1 with respect to the optical bandgap (crosses). The scattering of the points is important because of the oscillating nature of the overlap between the valence and conduction band states in the reciprocal space. Anyway, the main trend is a strong decrease of the recombination rate $1 / \tau$ with decreasing bandgap energy (it tends to zero for bulk silicon). We see that below $\sim 2.3 \mathrm{eV}$, the calculated radiative lifetimes are longer than $10 \mu \mathrm{s}$ in accordance with experimental data for the red band of porous silicon [2]. Therefore, we conclude that the red band of porous silicon can be interpreted by direct recombinations of electron-hole pairs in silicon nanostructures with dimensions between $2.5 \mathrm{~nm}$ and $4.5 \mathrm{~nm}$.

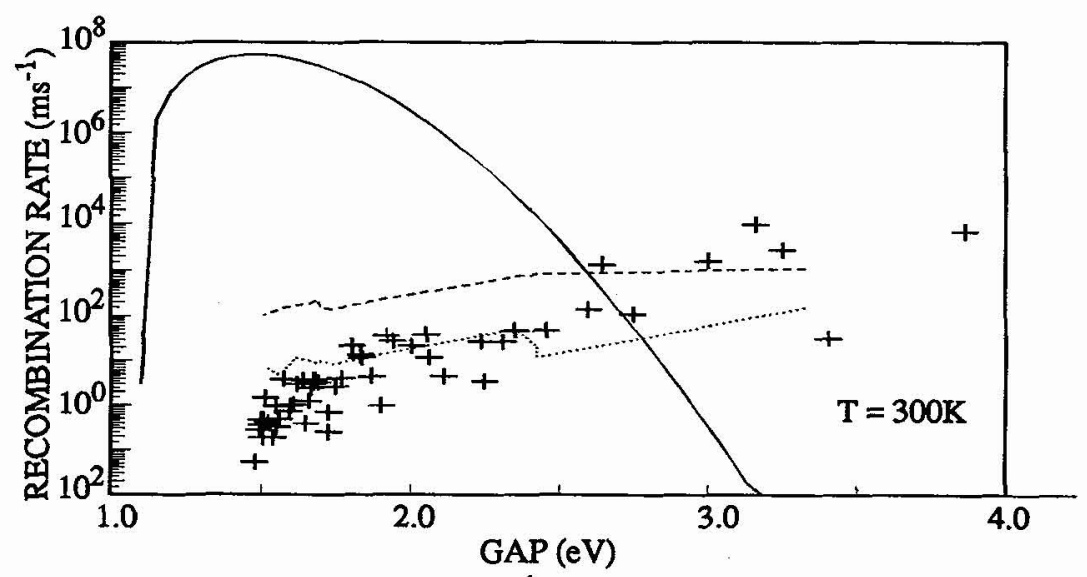

Fig. 2: Capture rates $(T=300 \mathrm{~K})$ of an electron or a hole in silicon crystallites due to a non radiative capture on a single neutral silicon dangling bond plotted with respect to the excitonic bandgap energy of the crystallite (continuous line). Crosses give the band to band radiative recombination rates of the electron-hole pairs in the same crystallites. The other curves are the radiative capture rates of carriers on a neutral dangling bond (hole capture: dashed line, electron capture: dotted line).

Concerning the infrared band, we have studied the influence of a dangling bond in the crystallites. On Fig. 1, dashed and dotted lines respectively represent the calculated radiative capture rates $(1 / \tau)$ of the hole and the electron on a neutral dangling bond as function of the bandgap of the crystallite. We see that the radiative capture by a dangling bond is a competitive process compared to the direct recombination. This could give rise to a luminescence with lower energy. However, it is well known that dangling bonds are very efficient non radiative recombination centers in bulk silicon (more specifically at $\mathrm{Si}-\mathrm{SiO}_{2}$ interfaces). The recombination is a two steps process. First, after excitation of an electron-hole pair, the hole or the electron is captured by the neutral dangling bond and, secondly, the remaining carrier is captured by the charged bond. We have calculated the probability of the first process for a non radiative capture (the radiative capture has been discussed above). This requires the determination of the capture cross sections in the crystallites. Cross sections are known experimentally for the $P_{b}$ center which corresponds to a dangling bond at $\mathrm{Si}_{-} \mathrm{SiO}_{2}$ interface [7]. However, we have shown that the cross sections strongly vary with the confinement. The thermal behavior of the cross section is usually approximated by: 


$$
\sigma=\sigma_{0} \exp \left(-\frac{E_{b}}{k T}\right), E_{b}=\frac{\left(E_{0}-d_{F C}\right)^{2}}{4 d_{F C}}
$$

with $E_{b}$ is the barrier height where $E_{0}$ is the ionization energy of the defect and $d_{F C}$ is the FranckCondon shift equal to the energy gain due to lattice relaxation after capture. The situation for the $P_{b}$ center is such that $E_{0} \sim d_{F C} \sim 0.3 \mathrm{eV}$ and the cross section is weakly thermally activated [7] (both for the capture of a hole or an electron). In crystallites, the situation is different because the ionization energy is enhanced due to confinement. In that case, the barrier for the recombination becomes important. But eqn. (2) is no longer valid for reasons exposed in ref. [7]. Instead of eqn. (2), we have used a recently derived analytic formula with a larger range of validity than eqn. (2). Therefore, using this formula and the experimental values of the capture cross sections, we have estimated the non radiative capture rates of the first carrier on a single dangling bond in the crystallites. Results are plotted on Fig. 1 with respect to the bandgap of the crystallites (continuous line). The recombination rate decreases at high energy due to the enhancement of the barrier and also decreases at low energy because the volume of the crystallite increases and the probability for a carrier to find a recombination center vanishes. We see that for crystallites with bandgap compatible with the red band of porous silicon $(\sim 1.4 \mathrm{eV}-2.2 \mathrm{eV})$ the non radiative capture by a dangling bond (continuous line) is much faster than the direct radiative recombination (crosses) or the radiative capture on the neutral dangling bond (dashed and dotted lines). Then the presence of one dangling bond in crystallite kills its "red luminescence".

We have also analyzed the capture of the remaining carrier. Because the ionization energy of the second capture is much larger than for the first one, the barrier becomes important $(\sim 0.5 \mathrm{eV})$ and the second multiphonon capture is improbable (a numerical estimation is difficult because experimental cross sections for the capture on charged dangling bonds are not known experimentally). Therefore, the second capture must be radiative, at least at low temperature. In a crystallite with bandgap equal to $1.7 \mathrm{eV}$, the energy of the emitted photon should be equal to $0.8 \mathrm{eV}$, in the infrared. So the infrared band of porous silicon can be coherently described by the recombination on dangling bonds in agreement with recent experimental results [3].

Recently, Calcott et al. [4] have shown that the exchange splitting in the lowest exciton state could explain the strong decrease of the luminescence intensity at low temperature. The exchange splitting is the difference between the energies of the $S=0$ and $S=1$ states where $S$ is the total spin of the electronhole pair. It has been proposed [4] that the exchange splitting, which is of the order of $\sim 0.1 \mathrm{meV}$ for the free exciton in bulk silicon, is greatly enhanced in crystallites due to quantum confinement. It is expected to be of the order of $\sim 10 \mathrm{meV}$. Therefore, at low temperature, only the $S=1$ state is populated and the lifetime is very long (in the millisecond range; it is not infinite because the spin-orbit interaction mixes the states together). Even if this explanation is reasonable, it is not sure that the exchange splitting is the only cause for the low temperature dependence of the intensity. In particular, Hybertsen et al. [8] and our group [6] have recently proposed that the valley orbit splitting could be of importance. In effect, these calculations show that there are several exciton states derived from degenerate band extrema which are split with an average spacing of about $10 \mathrm{meV}$. Note that these propositions have been challenged on the basis of Zeeman experiments [4]. To get a clearer idea of this complex problem, we have calculated the exchange splitting in the lowest exciton state. If the electron is in a state $\varphi_{c}$ and the hole in a state $\varphi_{v}$, it is easy to show that the exchange splitting $E_{x}$ is equal to:

$$
E_{x}=2 \int \frac{\varphi_{c}(r) \varphi_{v}(r) \varphi_{c}\left(r^{\prime}\right) \varphi_{v}\left(r^{\prime}\right)}{\varepsilon\left(r, r^{\prime}\right)\left|r-r^{\prime}\right|} d^{3} r d^{3} r^{\prime}
$$

where $\varepsilon\left(r, r^{\prime}\right)$ describes the screening in the crystallite. The states $\varphi_{c}$ and $\varphi_{y}$ are developed in the atomic basis. We only present preliminary results, calculations ar still in progress. For technical reasons, we have not estimated $E_{x}$ with the wave functions calculated using the previously described LCAO technique but with a much simpler tight-binding technique which by definition neglects the overlaps. Because the band structure in this method is not as good as using LCAO, bandgap energies can differ by $\sim 0.15 \mathrm{eV}$ $-0.25 \mathrm{eV}$. However, we believe that the following conclusions do not depend on this bandgap problem. Terms in the development of eqn. (3) in the basis of atomic orbitals are numerically computed using the form of the dielectric constant of ref. [9]. 


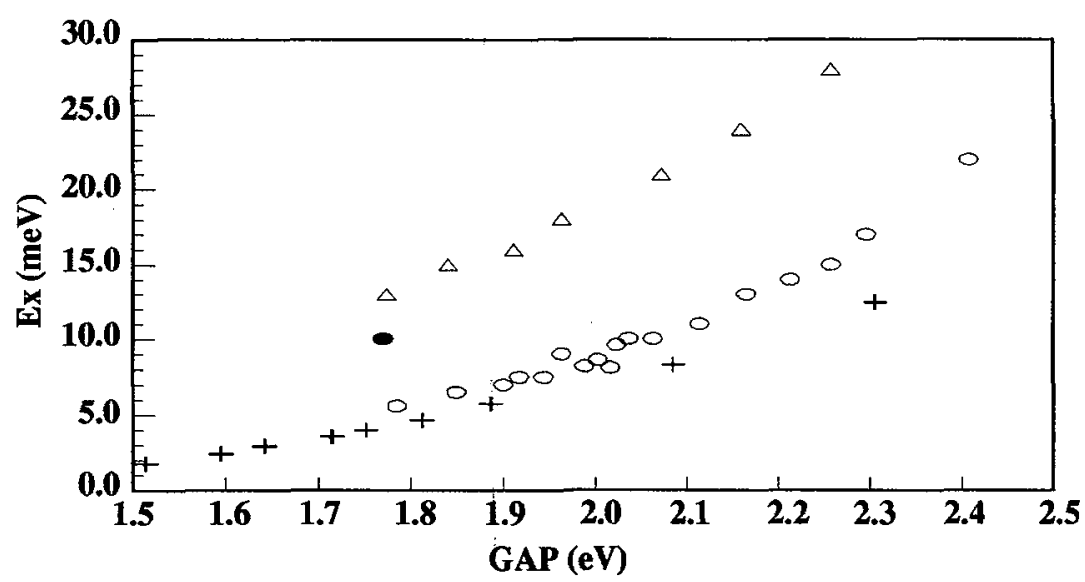

Fig. 2: Calculated exchange splitting (+) with respect to the optical bandgap of crystallites. We also plot the exchange splittings experimentally deduced from temperature dependence of the radiative lifetime (triangles) and from the onset observed in selectively excited photoluminescence (circles) [4].

The calculated exchange splittings are plotted in fig. 2 with respect to the bandgap of the crystallites (crosses). On fig. 2, we also plot the exchange splittings experimentally deduced from temperature dependence of the radiative lifetime (triangles) and from the onset observed in selectively excited photoluminescence [4]. The onset is interpreted by the fact that excitons are predominantly photocreated in the singlet $(S=0)$ than in the triplet state $(S=1)$ because of the difference in absorption strength of two or three orders of magnitude. We see that our results are quite close to the experimental data from excitation spectroscopy experiments but largely differ from those from temperature dependence of the radiative lifetime. The reason given in ref. [4] to explain the difference between the two experimental data sets is that there is a great variety of shapes and sizes of the confining structures which can have the same optical bandgap but can have a distribution of exchange splittings. Thermal experiments would measure the weighted average of the distribution whereas the onset of selectively excited photoluminescence would measure its minimum value. However, the difference between the two sets of data is large and it is not obvious that the distribution can be of this importance. Therefore, from our results, we can propose another explanation for the difference between the two data sets. The onset of selectively excited photoluminescence would measure the exchange splitting of the lowest exciton state - calculated here - but there are other exciton states above due to the valley-orbit interaction. The thermal experiments would measure an average between these states. Calculations are in progress to verify this interpretation. Obviously the compatibility of these results with Zeeman experiments should be checked [4].

In conclusion, calculations of electronic and optical properties of silicon crystallites show that the red and infrared light emissions of porous silicon are explainable by the quantum confinement plus the influence of dangling bonds. The exchange splitting of the lowest exciton state is calculated. The problem of the influence of the exchange and valley-orbit splittings on the luminescence lifetime remains an opened question.

[1] L.T. Canham, Appl. Phys. Lett. 57, 1046 (1990).

[2]J.C. Vial, A. Bsiesy, F. Gaspard, R. Hérino, M. Ligeon, F. Muller, R. Romestain, and R.M. Macfarlane, Phys. Rev. B 45, 14171 (1992).

[3]B.K. Meyer, D.M. Hofmann, W. Stadler, V. Petrova-Koch, F. Koch, P. Omling and P. Emanuelsson, submitted to Appl. Phys. Lett.

[4]P.D.J. Calcott, K.J. Nash, L.T. Canham, M.J. Kane and D. Brumhead, J. Phys: Condens. Matter. 5, L91 (1993).

[5]G . Allan, C. Delerue and M. Lannoo, Proceedings of the E-MRS 1993 Spring Meeting (Strasbourg May 4-7), Symposium on Light Emission from Silicon, to be published.

[6]J.P. Proot, C. Delerue and G. Allan, Appl.Phys, Lett. 61, 1948 (1992) and to be published.

[7]D. Goguenheim, and M. Lannoo, Phys. Rev. B 44, 1724 (1991).

[8]M.S. Hybertsen and M. Needels (to be published).

[9]G. Cappelini, R. Del Sole, L. Reining, F. Bechstedt, Phys.Rev. B 47, 9892 (1993). 